\title{
Effects of aging on the relationship between cognitive demand and step variability during dual-task walking
}

\author{
Leslie M. Decker • Fabien Cignetti • Nathaniel Hunt • \\ Jane F. Potter • Nicholas Stergiou • \\ Stephanie A. Studenski
}

Received: 22 November 2015 / Accepted: 22 July 2016/Published online: 3 August 2016

(C) American Aging Association 2016

\begin{abstract}
A U-shaped relationship between cognitive demand and gait control may exist in dual-task situations, reflecting opposing effects of external focus of attention and attentional resource competition. The purpose of the study was twofold: to examine whether gait control, as evaluated from step-to-step variability, is
\end{abstract}

L. M. Decker $(\bowtie)$

UMR-S 1075 COMETE (Mobilités: Attention, Orientation, Chronobiologie) INSERM and Université de Caen Normandie, Pôle des Formations et de Recherche en Santé, 2 rue des Rochambelles, 14032 Caen Cedex 5, France

e-mail: leslie.decker@unicaen.fr

L. M. Decker $\cdot$ N. Stergiou

University of Nebraska at Omaha, Biomechanics Research

Building, 6160 University Drive, Omaha, NE 68182-0860, USA

F. Cignetti

UMR 7291 CNRS and Aix-Marseille Université, 3 place

Victor-Hugo, 13331 Marseille Cedex 3, France

N. Hunt

Department of Integrative Biology, University of California, Berkeley, 3040 Valley Life Sciences Building \#3140, Berkeley, CA 94720-3140, USA

\section{J. F. Potter}

Division of Geriatrics and Gerontology, University of Nebraska Medical Center, 986155 Nebraska Medical Center, Omaha, NE 68198-6155, USA

S. A. Studenski

Translational Gerontology Branch, Longitudinal Studies Section, National Institute on Aging, 251 Bayview Blvd., Suite 100, Rm 04B316, Baltimore, MD 21224, USA related to cognitive task difficulty in a U-shaped manner and to determine whether age modifies this relationship. Young and older adults walked on a treadmill without attentional requirement and while performing a dichotic listening task under three attention conditions: nonforced (NF), forced-right (FR), and forced-left (FL). The conditions increased in their attentional demand and requirement for inhibitory control. Gait control was evaluated by the variability of step parameters related to balance control (step width) and rhythmic stepping pattern (step length and step time). A Ushaped relationship was found for step width variability in both young and older adults and for step time variability in older adults only. Cognitive performance during dual tasking was maintained in both young and older adults. The U-shaped relationship, which presumably results from a trade-off between an external focus of attention and competition for attentional resources, implies that higher-level cognitive processes are involved in walking in young and older adults. Specifically, while these processes are initially involved only in the control of (lateral) balance during gait, they become necessary for the control of (fore-aft) rhythmic stepping pattern in older adults, suggesting that attentional resources turn out to be needed in all facets of walking with aging. Finally, despite the cognitive resources required by walking, both young and older adults spontaneously adopted a "posture second" strategy, prioritizing the cognitive task over the gait task.

Keywords Aging · Gait · Cognitive control $\cdot$ Step variability $\cdot$ Resource competition 


\section{Introduction}

Aging is associated with decrements in both executive functions, also called executive control or cognitive control (Diamond 2013), and gait control as evaluated from the variability of gait patterns (Buzzi et al. 2003; Owings and Grabiner 2004; Kang and Dingwell 2008). In older adults, interference caused by competing demands for attentional resources has been demonstrated when gait is performed simultaneously with a cognitive task (i.e., dual-task paradigm). Studies have reported either increased step-to-step variability of gait parameters (e.g., step velocity, step time, and step length) or cognitive performance decrements under dual-task conditions (Beauchet et al. 2005a; Dubost et al. 2006; Hollman et al. 2007). The decline in performance of either gait or cognitive processing relative to either task performed alone gives evidence that the concurrent tasks compete for attentional resources with aging. Importantly, the dual-task-related changes in gait variability were found to be predictors of falls in older adults (Kressig et al. 2008; Herman et al. 2010). However, not all studies reported such age-related interference effects in dualtask walking (Yogev et al. 2005; Springer et al. 2006). A mixed pattern of findings has also been seen in healthy young adults. Some studies showed no dual-task-related changes in gait variability, suggesting that the regulation of gait patterns proceeds without cognitive control (Beauchet et al. 2005b), while others found either detrimental (i.e., increased gait variability) or beneficial (i.e., decreased gait variability) effects (Grabiner and Troy 2005; Dubost et al. 2008). Thus, the association between cognitive control and gait variability and its changes with aging appear complex. Providing a model framework for these issues is important for future studies and for developing interventions that may reduce the negative impacts of dual tasking on fall risk.

A dual-process account of cognitive-motor interactions including opposing effects of "external focus of attention" and "attentional resource competition" on gait variability may account for the above mixed findings (Lövdén et al. 2008; Verrel et al. 2009). Simple cognitive tasks would shift the focus of attention away from gait control, thus reducing top-down cognitive (or attentional executive) control of gait (i.e., decreased gait variability). Indeed, low variability often indicates processes that proceed with little cognitive control (Newell and Corcos 1993; Hausdorff 2005). Inversely, higher levels of cognitive task difficulty would alter gait control (i.e., increased gait variability) through attentional resource competition ( $\mathrm{Li}$ and Lindenberger 2002; Schaefer et al. 2006). The two processes would trade off at lower levels of task difficulty in older adults, presumably as a result of age-related increased reliance on higher-level cognitive processes for gait control (i.e., less automaticity), coupled with reduced cognitive control efficiency (Seidler et al. 2010). However, empirical support for this model remains insufficient. Studies either failed to detect a clear trade-off between the two processes with increasing cognitive control demand in older adults (Lövdén et al. 2008) or reported such tradeoff using gait parameters lacking of functional significance (Verrel et al. 2009).

Accordingly, the objective of the present study was to test the likelihood of the dual-process account (external focus/resource competition) relating cognitive control demand and gait variability and determine whether the negative effects of attentional resource competition on gait variability become more pronounced as we get older. Cognitive control is often invoked by environmental stimuli that are perceptually salient or that trigger default action tendencies (Braver and Cohen 2000; Braver et al. 2002; Miller and Cohen 2001). The classic and probably most widely known example of a stimulus situation that imposes cognitive control is the Stroop task (Stroop 1935) with the presentation of color words written in a conflicting ink (e.g., the word "red" written in a blue ink). In the ink color naming condition, a default action tendency is to respond with the color word even when the instruction is to respond with the ink color. This phenomenon, called the "Stroop effect," has thus a "built-in" perceptual bias since the semantic information is perceptually more salient than the nonsemantic one. Thought, a limitation in the classical approach to cognitive control is that the conflict is either present (i.e., "read the ink color") or absent (i.e., "read the color word"). Hence, a fine-grained analysis of agerelated differences in cognitive control would not be possible. To address this issue, Hugdahl et al. (2009) proposed a dichotic listening task allowing a parametric manipulation of the degree of cognitive conflict and a corresponding quantification of the degree of cognitive control needed to resolve the conflicting information. Literally, dichotic listening means that two different auditory stimuli (i.e., simple speech sounds like consonant-vowel syllables) are presented, one to the right ear and one to the left ear, at the same time (Bryden 1988; Hugdahl 2003; Tervaniemi and 
Hugdahl 2003). In a non-forced (NF), free recall, condition (i.e., participants report the syllable they hear best with no instruction on focus of attention), a preference for the syllables given to the right ear over those given to the left ear is normally found in healthy adults. There are at least three explanations for this ear bias. The structural hypothesis suggests that the right ear projects more strongly to the language dominant left hemisphere (Kimura 1961). The callosal relay hypothesis is based on the influence of inhibitory connections via the corpus callosum (Asbjørnsen and Hugdahl 1995). The attentional hypothesis suggests that each hemisphere primarily directs attention to contralateral space and because the left hemisphere is dominant for processing speech stimuli, attention is primarily directed to the right ear (Kinsbourne, 1970, 1975). However, by instructing participants to attend exclusively to one ear [forced-right (FR) or forced-left (FL)], the right ear advantage (REA) can be modulated, that is, top-down (instruction-driven) attentional modulation of the bottom-up (stimulusdriven) lateralized perceptual REA effect is obtained. Typically, the REA is increased in the FR condition, and decreased, or switched to a left ear advantage (LEA), in the FL condition (Bryden et al. 1983; Hugdahl and Andersson 1986). Hence, humans have the ability to overcome a default, stimulus-driven, REA by switching attention from one ear to the other. This can be seen as a rough, experimental, analogue to the well-known "cocktail party phenomenon," which also involves the use of attention in switching between multiple speech inputs (Cherry 1953).

Besides its ecological validity, the strength of the dichotic listening task is twofold in the context of the dual-process account of cognitive-motor interactions (Huxhold et al. 2006; Lövdén et al. 2008; Schaefer et al. 2015; Verrel et al. 2009). First, the cognitive demand and effort parametrically increase from the NF to the FL condition (Hugdahl et al. 2009). In the NF condition, attention is attracted in a non-volitional way (i.e., bottom-up) to the right ear. Inversely, attentional processing becomes volitional (i.e., top-down) in the FR and FL conditions, leading to further cognitive effort compared with the NF condition. Moreover, greater volitional, or intentional, control is required in FL due to antagonistic actions of the top-down and bottom-up processes, with the bottom-up process pushing for a right ear response and the top-down process pushing for a left ear response. This is in contrast to the FR condition where the two processes act synergistically, both pushing for a right ear response. To summarize, although the auditory stimuli are identical across conditions, the three different instructional conditions allow teasing apart a lateralized perceptual process (NF condition), an orienting process with synergistic bottom-up and top-down effects (FR condition), and a cognitive control process with a conflicting top-down effect that needs to override the bottom-up, automatic response. The NF condition in this context serves as a baseline condition to evaluate the effects in the FR and FL conditions (Hugdahl et al. 2009). Second, inhibitory control is involved in the two forced attention conditions to prevent the irrelevant stimulus from gaining access to the focus of attention, with increasing requirement for that control from FR to FL (Della Penna et al. 2007; Hugdahl et al. 2009). Functional magnetic resonance imaging (fMRI) studies have confirmed additional recruitment of cognitive control to resolve the interference in the FL condition as compared to the FR condition, showing remaining significant activations in the frontal and anterior cingulate cortex, when contrasting fMRI images obtained during the FL condition with those obtained during the FR condition (Thomsen et al. 2004; see also Hugdahl et al. 2009 and references therein). Considering that cognitive control requires intact frontal lobes, and particularly the dorsolateral and ventral parts of the prefrontal cortex (Braver et al. 2002), as well as the dorsal part of the anterior cingulate (Bush et al. 2000), it has been argued that the FR and FL conditions differ in the degree to which they involve cognitive control and specifically inhibitory control. Given that inhibitory processes play an important role in balance control, especially in older adults (Redfern et al. 2001, 2009), dichotic listening is likely to promote attentional resource competition with gait. As regards to walking, our analysis focused on step-to-step variability, including spatial (step length and step width variability) and temporal (step time variability) variability parameters. Increments in these parameters have been consistently associated with both impaired gait control and higher risk of falls among older adults (Hausdorff et al. 2001; Brach et al. 2005; Verghese et al. 2009), as well as increased reliance on attention and cognitive control (Hausdorff 2005).

On the basis of the dual-process account relating cognitive control to gait variability (Lövdén et al. 2008), we hypothesized: (i) no dual-task-related changes (i.e., recall accuracy and modulation of the ear advantage, assessed by the number of errors and the 
laterality index, respectively) in dichotic listening performance; (ii) a decreased variability of step width, step time, and step length in the NF condition (external focus of attention) and an increasing variability from the NF through the FR to the FL condition (resource competition); and (iii) further increases in step parameter variability from the NF through the FR to the FL condition (larger resource competition) in older adults due to more limited attentional resources with aging.

\section{Methods}

Study population

Young adults $(n=20)$ aged between 20 and 35 years and older adults $(n=19)$ aged 65 years or older were recruited for the experiment. The two samples matched regarding their demographic characteristics (Table 1). Inclusion criteria were (1) native English speakers; (2) right-handed (Edinburgh Handedness Inventory; Oldfield 1971; see also Foundas et al. 2006, who demonstrated that, in verbal dichotic listening, right- and left-handers differ in the size of the shift in laterality when attention is directed to either the right or left ear); (3) living independently in the community; (4) able to ambulate without the use of, or assistance from, a prosthetic device, a fixed or mobile walking frame, or other assistive devices (e.g., brace, cane, crutch) or without the assistance of another person; and (5) not diagnosed with neurologic conditions (e.g., Alzheimer's disease, Parkinson's disease, stroke, and multiple sclerosis) or other conditions (e.g., dementia, moderate or severe chronic obstructive pulmonary disease, weight-bearing pain, chest pain at rest or during activity, previous history of myocardial infarction, dyspnea at rest, or use of supplemental oxygen). Exclusion criteria were (1) a hearing level too poor to perceive speech (i.e., a threshold higher than $20 \mathrm{~dB}$ at $2 \mathrm{kHz}$ ) and (2) inter-aural, puretone threshold difference exceeding an average of $10 \mathrm{~dB}$ in the speech range during audiometric evaluation (hearing differences of $10 \mathrm{~dB}$ between ears are found to affect dichotic listening performance, Asbjørnsen et al. 2000). Thus, all participants underwent an audiometric test performed for each ear at .25, .5, 1, 2, 4, and $8 \mathrm{kHz}$, with testing repeated at $1 \mathrm{kHz}$. Informed consent was obtained from all participants prior to data collection according to the guidelines of the university's institutional review board.

\section{Protocol overview}

The experiment took place in the Nebraska Biomechanics Core Facility (Omaha, NE). It consisted of a sitting session and a walking session, counterbalanced between participants. In the sitting session, the participants underwent

Table 1 Baseline characteristics of the adult participants

\begin{tabular}{|c|c|c|c|c|c|c|}
\hline & & Young & & Older & & Statistics \\
\hline \multirow[t]{5}{*}{ Demographics } & Gender $(\mathrm{M} / \mathrm{F})$ & $8 / 12$ & & $10 / 9$ & & $x^{2}(1, N=39)=.63 ; p=.43$ \\
\hline & Age (years) & $24.45(.87)$ & {$[20-35]$} & $69.26(1.11)$ & {$[65-79]$} & $t(37)=-31.96 ; p<.0001$ \\
\hline & Height (m) & $1.73(.02)$ & {$[1.6-1.86]$} & $1.71(.02)$ & {$[1.57-1.91]$} & $t(37)=1.05 ; p=.29$ \\
\hline & Body mass (kg) & $70.41(2.63)$ & {$[52-94]$} & $77.45(2.78)$ & {$[59-105]$} & $t(37)=-1.83 ; p=.07$ \\
\hline & Education (years) & $16.5(.43)$ & {$[11-20]$} & $17.63(.82)$ & [13-24] & $t(37)=-1.22 ; p=.23$ \\
\hline \multirow[t]{4}{*}{ Cognition } & DS forward & $11.78(.61)$ & {$[7-16]$} & $10.05(.48)$ & {$[7-14]$} & $t(37)=1.79 ; p=.08$ \\
\hline & DS backward & $7.6(.47)$ & {$[4-13]$} & $6.47(.56)$ & {$[2-11]$} & $t(37)=1.89 ; p=.06$ \\
\hline & DS total & $19.47(.94)$ & [12-29] & $16.52(.93)$ & {$[10-23]$} & $t(37)=1.96 ; p=.04$ \\
\hline & MMSE & $29(.21)$ & {$[27-30]$} & $28.94(.32)$ & {$[26-30]$} & $t(37)=.13 ; p=.9$ \\
\hline Depression & GDS & & & $1.21(.32)$ & {$[0-4]$} & \\
\hline Fear of falling & MFES & & & $9.94(.04)$ & {$[9-10]$} & \\
\hline Gait & PWS $\left(\mathrm{m} \mathrm{s}^{-1}\right)$ & $1.06(.03)$ & {$[.72-1.43]$} & $.77(.04)$ & {$[.45-1.02]$} & $t(37)=4.81 ; p<.0001$ \\
\hline
\end{tabular}

Entries are mean (standard error) [range]

$D S$ digit span, MMSE Mini-Mental State Examination, GDS Geriatric Depression Scale, MFES Modified Falls Efficacy Scale, PWS preferred walking speed 
neuropsychological testing. Then, they performed the dichotic listening task while seated in order to establish baseline cognitive performance. In the walking session, they walked on a treadmill at their preferred walking speed (Table 1), either without an explicit attentional requirement (i.e., control walking condition) or while performing the three conditions (NF, FR, and FL) of the dichotic listening task (i.e., dual-task conditions). Each session lasted $1 \mathrm{~h}$ and $30 \mathrm{~min}$, with a 1-week interval between sessions.

\section{Neuropsychological test battery}

The participants first underwent tests of general cognitive functioning, involving the Mini-Mental State Examination (see Folstein et al. 1975 for normative scores) and the Wechsler Adult Intelligence ScaleThird Edition (WAIS-III) forward and backward digit span tests (Wechsler 1981; see Monaco et al. 2013 for normative scores). Further, baseline data with respect to the number of falls in the year prior to the experiment, fear of falling (Modified Falls Efficacy Scale; Hill et al. 1996), and depression (15-item Geriatric Depression Scale; Sheikh and Yesavages 1986) were obtained for the older adults.

\section{Dichotic listening task}

The auditory stimuli in the English version of the standard Bergen dichotic listening paradigm (Hugdahl and Andersson 1986; Hugdahl 2003) were phonetically meaningful but semantically meaningless consonant-vowel (CV) syllables (/ba/, /da/, /ga/, $/ \mathrm{pa} /, / \mathrm{ta} /, / \mathrm{ka} /$ ). The six CV syllables were combined in pairs in all possible combinations, thus forming a total of 36 possible dichotic pairs, including the 6 homonymic pairs (da-da, etc.). The homonymic pairs were used as a perceptual control to ensure that participants were able to perceive the different CV syllable stimuli (i.e., three out of six were considered adequate); however, they were not included in the statistical analysis. Thus, the maximum score was 30 for each ear. In each trial, one CV syllable was presented to the left ear and another simultaneously to the right ear. Each CV syllable was originally read by a male voice with intonation and intensity held constant. Each CV syllable pair was recorded three times, with three different randomizations of the 36 dichotic pairs. Thus, the total number of trials was 108 . The 108 trials were divided into three blocks of 36 trials, one block for each instructional condition (NF, FR, and FL). Each CV syllable stimulus was approximately 480$550 \mathrm{~ms}$ in duration, and the inter-stimulus interval was approximately $4000 \mathrm{~ms}$. The total duration of each condition was set at $3 \mathrm{~min}$.

A standard PC running the E-prime programming platform (www.pstnet.com; Psychology Software Tools) was used for stimulus presentation and response collection. Oral responses were also recorded using a digital voice recorder as back-up and analyzed further. Before the dichotic listening task was performed, the participants were shown the six syllables written in large font on a piece of paper placed in front of them and were asked to name them. This was done to ensure that the participants were familiar with the syllables and their correct pronunciation. The stimuli were presented via noise-cancelling headphones. The audio output from the computer was monitored by the experimenter through an extra set of headphones placed only over one ear in order to follow the progress of the test.

The test administration and scoring procedure were the same as those of Hugdahl and Andersson (1986). The dichotic listening task was divided into three different conditions, which differed with regard to the instruction on how to focus attention. In all three conditions, the participants were told that they would be presented with a list of CV syllables and that their task was to answer with the syllable they heard on each trial. Before the actual testing, a few practice trials were presented, and the participants were instructed to report whether they heard the CV syllables and to give feedback about whether the audio level was loud enough. In the NF condition, participants were told to report after each trial the single CV syllable which they heard best or most clearly from the six possible syllables. In the FR and FL conditions, they were asked to listen to and report the syllables from the right or left ear, respectively, and to ignore any sounds they might hear in the other ear. In order to make sure that the participant had understood which ear to attend to during the FR and the FL conditions, the experimenter touched or pointed the ear of the participant on that side. The NF condition was always presented first to avoid that prior execution of either of the forced conditions produced carryover effects, with the risk of biasing attention toward the previously attended ear. The presentation order of the two forced 
conditions was counterbalanced across participants (either FR before FL or FL before FR).

\section{Gait task}

Prior to data collection, reflective markers were attached to a tight-fitting suit at specific anatomical landmarks of each participant's lower limbs (Nigg et al. 1993; Vaughan et al. 1999). The anatomical landmarks were the anterior and posterior superior iliac spine, lumbosacral joint, greater trochanter of the femur, lateral midthigh, front lower thigh, lateral and medial epicondyles of the femur, front mid-shank, lateral lower shank, lateral and medial malleoli, lateral border of the fifth metatarsal head, medial border of the first metatarsal head, lateral and medial processes of the calcaneal tuberosity, heel, and between the second and third metatarsophalangeal joints. The three-dimensional positions of the markers were collected at $100 \mathrm{~Hz}$ with a Motion Analysis Eagle Digital system (eight cameras), which was interfaced with the EVaRT software (version 5.0, Motion Analysis Corporation, Santa Rosa, CA).

All participants were fitted into the LiteGait ${ }^{\circledR}$ (Mobility Research, LLC, Tempe, AZ) harness system for safety. The LiteGait ${ }^{\circ}$ supported the participants only if balance was lost during testing. Participants were given ample time to familiarize themselves with walking on the treadmill prior to testing. The preferred walking speed (PWS) was established using a wellestablished protocol (Jordan et al. 2007). Initially, the participants walked at a relatively slow speed, and then the investigator increased the speed in $.1 \mathrm{~km} \mathrm{~h}^{-1}$ increments until the participants reported their PWS. The speed was then increased by approximately $1.5 \mathrm{~km} \mathrm{~h}^{-1}$ and then decreased by $.1 \mathrm{~km} \mathrm{~h}^{-1}$ until the PWS was reestablished. This procedure was repeated until a close match was achieved (less than $.4 \mathrm{~km} \mathrm{~h}^{-1}$ difference). While establishing their PWS, participants were prevented from viewing the treadmill speed display. Afterwards, participants walked either without an explicit attentional requirement (i.e., control walking condition) or while performing the three conditions (NF, $\mathrm{FR}$, and FL) of the dichotic listening task (i.e., dual-task conditions). No specific instructions were given about task prioritization under dual-task conditions. For each condition, participants walked for 3 min and ample rest was provided between conditions. The duration of $3 \mathrm{~min}$ was chosen as it is difficult to maximize concentration or sustained attention for a longer period of time (Hunt and Lansman, 1986; Aylward et al. 1997; Griggs et al. 1998). The order of the walking conditions was counterbalanced between participants.

\section{Assessment of dichotic listening performance}

A response was counted as correct when the recalled syllable matched the syllable presented to either the right (RE) or left (LE) ear on each trial; if the response did not match either syllable, or no response was given, it was counted as an error. The number of errors (Err) was calculated as follows:

$\mathrm{Err}=30-(\mathrm{RE}+\mathrm{LE})$

The laterality index (LI) was calculated to quantify the ear advantage using this formula:

$\mathrm{LI}=\left[\frac{(\mathrm{RE}-\mathrm{LE})}{(\mathrm{RE}+\mathrm{LE})}\right] \times 100$

The laterality index thus ranged between $+100 \%$ (maximum REA) and $-100 \%$ (maximum LEA). Values close to $0 \%$ indicate no ear advantage. In the NF condition, a $10 \% \mathrm{LI}$ is within the normal range found in healthy right-handers (Hugdahl 2003). Attention clearly modulates the direction and degree of ear asymmetry in dichotic listening. A reduction in the ability to modulate the ear advantage is typically observed with advancing age when instructed to focus attention on and report the left ear syllables. As a matter of fact, older adults fail to report more syllables from the left ear during the FL condition (Hugdahl et al. 2009). This may be attributed to a breakdown of the executive attention network, which is mediated by frontal (inhibitory control of attention) and parietal (spatial selective attention and "disengagement processes" to switch attention from one ear to the other) regions. It has been suggested that interhemispheric (collosal atrophy) as well as intrahemispheric (subcortical white matter lesions) disconnectivity contributes to an age-related increased difficulty focusing attention on the left ear (Bouma and Gootjes 2011).

\section{Assessment of step variability}

The marker trajectories were low-pass filtered at $10 \mathrm{~Hz}$ with a zero-lag Butterworth filter. Step length and step time calculation required first identifying toe-off events from the maximum backward displacements of the 
(antero-posterior) x-coordinates of the toe markers (i.e., right and left markers located between the second and third metatarsal phalangeal joints). Step length was afterwards determined as the horizontal distance between the positions of the toe markers at each toe-off event, and step time was obtained as the time interval between consecutive toe-off events (Decker et al. 2012, 2013). Step width required first calculating the right and left foot midpoints. The foot midpoint was defined as the midpoint of the vector connecting the heel marker to the toe marker. Step width was afterwards determined as the medio-lateral distance between the foot midpoints calculated over consecutive foot-flat instants. Specifically, the right (or left) foot-flat instants were identified when the left (or right) swing limb's knee passed in front of the right (or left) stance limb's knee, using the (anteroposterior) $\mathrm{x}$-coordinates of the markers located on the lateral epicondyles of the femurs. For consistency across subjects, step time, step length, and step width time series were shortened to 256 data points, which was the number of consecutive steps collected from the slowest subject. All step parameters were calculated using MATLAB (The MathWorks, Inc., MA, version 7.0).

Step-to-step variability was afterwards determined from the standard deviation of the time series instead of the coefficient of variation following previous recommendations (Lövdén et al. 2008). First, the need for normalizing to the mean was not necessary given that our central hypothesis focused on an age $\times$ cognitive demand interaction effect (i.e., looking for a greater increase in step parameter variability with cognitive demand in older adults compared to their younger counterparts) rather than on a main effect of age. Second, normalizing the standard deviation with the mean would have been meaningful in case an age $\times$ cognitive demand interaction effect also applies for the estimates of the mean step parameters, which was neither predicted nor empirically found. Accordingly, we stayed closer to the raw data and avoided relying on the normalization assumptions involved when computing the coefficient of variation (e.g., mean has priority over variability).

\section{Statistical analysis}

The gait (standard deviations of step length, step time, and step width) and cognitive-dichotic listening (laterality index, number of errors) dependent variables displayed acceptable normal distribution as assessed using the Kolmogorov-Smirnov test. The gait variables were examined using two separate mixed-design analyses of variance (ANOVAs). A 2 (group: young adults, older adults) $\times 2$ (condition: walking, walking $+\mathrm{NF}$ ) ANOVA addressed the effect of the low level of attentional demand on step variability. A 2 (group: young adults, older adults) $\times 3$ (condition: walking $+\mathrm{NF}$, walking + FR, walking + FL) ANOVA addressed the effect of increasing attentional demand on step variability. Given that slower speeds in the older adults may confound the effect of age on step variability (e.g., Kang and Dingwell 2008), we also adjusted the step variability dependent variables by the group means of the PWS, thereby running analyses of covariance (ANCOVAs) with PWS as the covariate (see for instance Cignetti et al. 2012 for a similar approach). For dichotic listening performance, we performed a 2 (group: young adults, older adults) $\times 2$ (session: sitting, walking) $\times 3$ (condition: NF, FR, FL) ANOVA. When the assumption of sphericity was violated for the within-subject effect (as evaluated using Mauchly's test), the degrees of freedom (reported truncated to the nearest integers) and thereby the $F$ value were corrected using the GreenhouseGeisser correction in all above analyses. Post hoc multiple comparisons were conducted using the NewmanKeuls test. Effect sizes are reported as partial etasquared $\left(\eta^{2}\right)$. Statistical significance was set at .05 . Statistica (version 10, Statsoft, Inc., Tulsa, OK, USA) was used to perform all analyses.

\section{Results}

Baseline characteristics of the young and older adults

All participants scored more than 25 on the MMSE and more than 6 and 5 on the WAIS-III forward and backward digit span tasks, respectively, which reflected preserved general cognitive function (Table 1). Moreover, the older adults reported no falls in the year prior to the experiment, had no fear of falling (i.e., MFES scores close to 10), and had an average score very close to 1 on the 15-item GDS, indicating no symptoms of depression.

\section{Dichotic listening performance}

Mixed-design ANOVA conducted on the laterality index revealed a significant condition main effect $\left(F[1,45]=26.72, p<.001,{ }^{2}=.39\right)$ and a group $\times$ 
condition interaction effect $(F[1,45]=8.30$, $p=.003,{ }^{2}=.12$ ). In young adults, the laterality index significantly increased from the NF to FR conditions and significantly decreased from the FR to FL conditions, reaching negative values in the latter condition (Fig. 1). As expected, young adults showed REA in NF, increased REA in FR, and LEA in FL. The trend was less pronounced in older adults, which explained the interaction effect. Post hoc tests did not reveal any significant differences between the three conditions, meaning that older adults failed to modulate the REA. With respect to the number of errors, there was a significant group main effect $\left(F[1,34]=22.77, p<.001,{ }^{2}=.41\right)$, with significantly larger values in older adults as compared to young adults (Fig. 1). Finally, the mixed-design ANOVA conducted on the number of errors revealed a group $\times$ session interaction effect $\left(F[1,34]=4.76, p=.036,{ }^{2}=.12\right)$. This effect was explained by the fact that young adults made more errors, whereas older adults made fewer errors while walking than while seated.
Step variability

ANOVAs that evaluated the effect of the lowest level of attentional demand in dichotic listening (NF condition) on step variability revealed a significant group main effect for step length $\left(F[1,37]=7.07, p=.01, \eta^{2}=.16\right)$ and step time $\left(F[1,37]=22.51, p<10-5, \eta^{2}=.38\right)$, with a lower variability (i.e., lower standard deviation values) in the young adults than in the older adults (Fig. 2). There was also a significant condition main effect for the variability of step length $(F[1,37]=12.98, p<.001$, $\left.\eta^{2}=.26\right)$, step time $\left(F[1,37]=7.33, p=.01, \eta^{2}=.16\right)$, and step width $\left(F[1,37]=6.39, p=.01, \eta^{2}=.15\right)$. For all condition effects, post hoc analyses showed decreased variability in the walking + NF condition as compared to the control walking condition. ANCOVAs run (with PWS as the covariate) on step variability dependent variables only partially confirmed the above results. There remained a significant decreased variability in step length $\left(F[1,37]=7.85, p<.001, \eta^{2}=.18\right)$ and step width $\left(F[1,37]=6.39, p=.01, \eta^{2}=.15\right)$ and a marginally significant decrease in step time variability $(F[1,37]=3.07$,
Fig. 1 Mean (standard error) dichotic listening performance as evaluated from the laterality index and the number of errors. For both young and older adults, performance is reported as a function of dichotic listening condition ( $N F$ nonforced, $F R$ forced-right, $F L$ forced-left) and session (ST single-task, $D T$ dual-task)
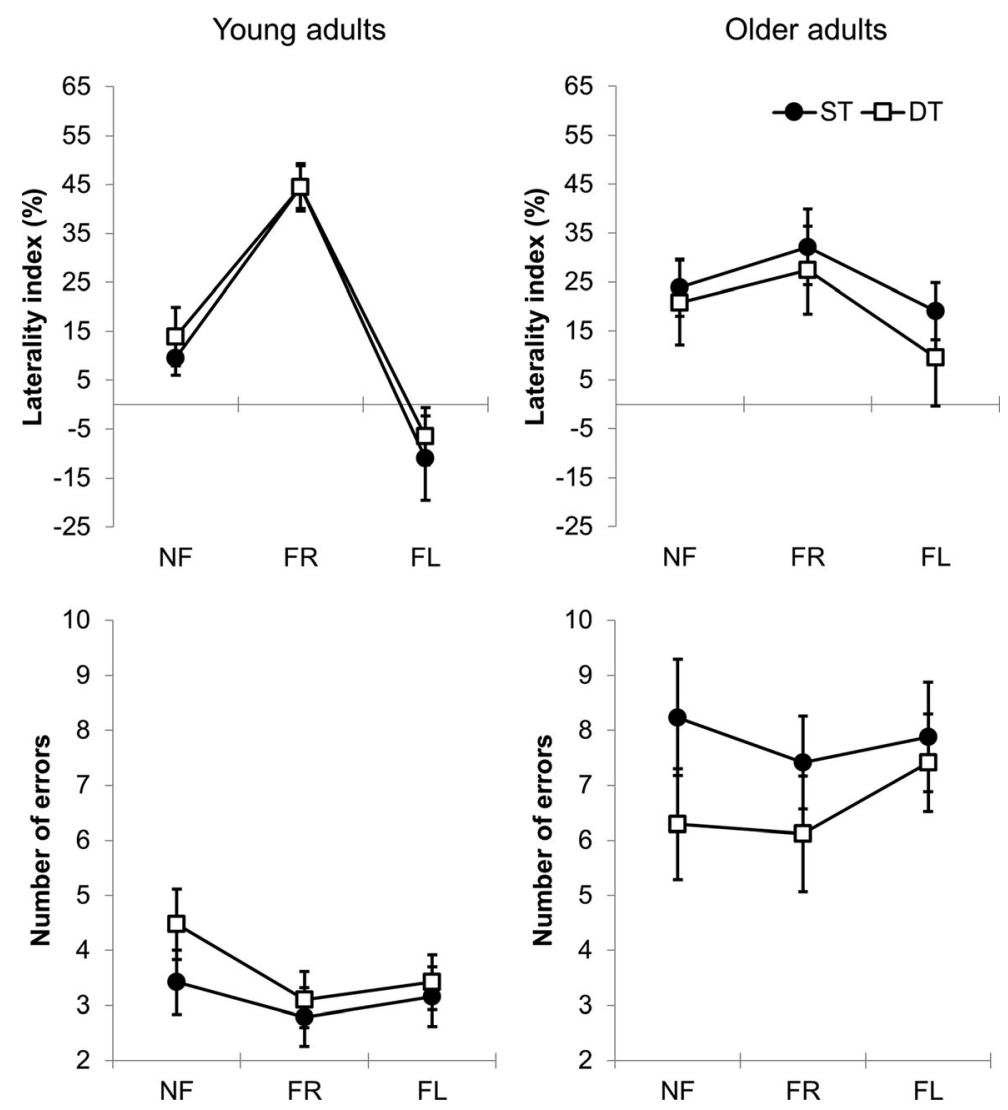


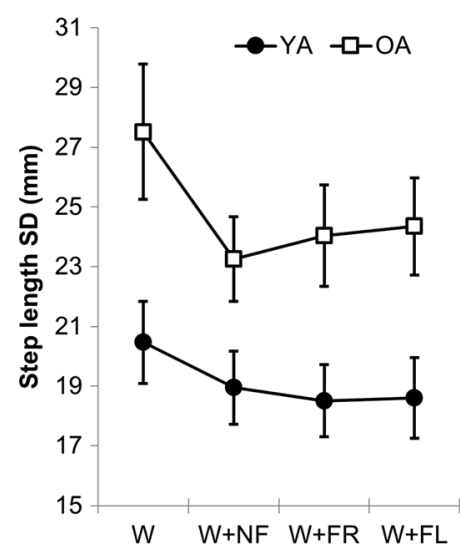

Fig. 2 Changes in mean (standard error) values of step length, step time, and step width variability with age group and condition. Age group includes young adults $(Y A)$ and older adults $(O A)$.

$p<.01, \eta^{2}=.08$ ) from the walking condition to the walking $+\mathrm{NF}$ condition (i.e., condition main effect), while the group main effects for step length, step time, and step width variability were no longer significant. Therefore, increased variability in older adults resulted from slower walking speeds.

ANOVAs that addressed the effect of increasing attentional demand in dichotic listening on variability yielded a significant group main effect for step length $\left(F[1,37]=7.54, p<.01, \eta^{2}=.17\right)$ and step time $(F[1$, $\left.37]=18.91, p<.001, \eta^{2}=.34\right)$, with a lower variability in the young adults than in the older adults (Fig. 2). The analyses also revealed a significant condition main effect for step time variability $(F[2,69]=3.43, p=.04$, $\left.\eta^{2}=.1\right)$ and step width variability $(F[2,68]=5.52$, $\left.p=.006, \eta^{2}=.13\right)$, whose values were larger in the walking + FL condition than in the walking $+\mathrm{NF}$ condition. Inspection of the data (Fig. 2) showed that such increasing trend from walking $+\mathrm{NF}$ condition to walking + FL condition applied to both young and older adults with regards to step width variability, while it was mainly driven by the older adults regarding step time variability. Although the group $\times$ condition interaction was not found to be significant for step time variability, one-way repeated measures ANOVAs run separately on the young adult and the older adult group data confirmed our intuition, with a significant increase of step time variability in the older adults only $(F[2,36]=3.32$, $p=.04, \eta^{2}=.15$ ). Finally, the group main effect for step length and step time variability disappeared using ANCOVAs (i.e., the greater variability in the older adults resulted from their slower speeds), while the condition main effect for step time variability $(F[2$,

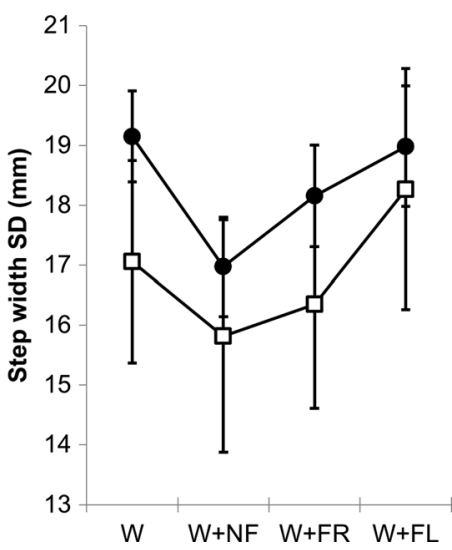

Condition includes walking alone $(W)$ and walking while performing dichotic listening in non-forced $(W+N F)$, forcedright $(W+F R)$, and forced-left $(W+F L)$

$\left.69]=3.23, p=.04, \eta^{2}=.07\right)$ and step width variability $\left(F[2,68]=5.52, p=.006, \eta^{2}=.13\right)$ remained significant.

\section{Discussion}

The present experiment examined age-related changes in the relationship between gait control, as evaluated by step-to-step variability, and the difficulty of a cognitive task involving inhibitory control. The results are twofold. First, a U-shaped relationship was observed in young and older adults with respect to step width variability, with a reduced variability in the NF condition and an increasing variability from the NF to the FL conditions. Second, step time variability was also related to attentional demand in a U-shaped manner in the older adults, with the turning point at NF, while step length variability decreased in NF but leveled out for higher attentional demands. Inversely, variability in both step time and step length was not modulated by attentional demand in the young adults. Therefore, a Ushaped relationship was observed for variability in both step width and step time in the older adults and for step width variability only in the young adults, supporting a dual-process account relating cognitive demand and gait control in both groups. Low attentional demand (NF condition) improved gait control (i.e., decreased variability), likely shifting the focus of overt attention away from the walking task. Higher demands (forced attention conditions) overshadowed this beneficial effect, hindering gait control (i.e., increased variability) through attentional resource competition. However, 
although these findings support the dual-process account (Lövdén et al. 2008; Verrel et al. 2009), the fact that step variability in the most cognitively demanding FL condition did not exceed that in the control walking condition is counterintuitive in the framework of attentional resource competition. An explanation may be that walking alone already required attentional resources to not walk off the treadmill, the person regulating his/her gait by limiting deviations from a "preferred operating point" (Dingwell et al. 2010; Decker et al. 2012; Dingwell and Cusumano 2015). Because of this control strategy and given that treadmill walking imposes boundaries on the extent of deviations from one step to the next, exceeding a ceiling value (both in the antero-posterior and mediolateral directions) might have not been possible in the FL condition. Another explanation may have to do with a too low degree of interference between cognitive processing in the FL condition and gait control. However, this is unlikely given that the degree of cognitive demand is considered to be high in that condition (Hugdahl et al. 2009). Moreover, the demand in the FL condition is on inhibitory control, which is also largely involved during walking through sensory integration processes for balance control (Redfern et al. 2001, 2009).

Previous investigators suggested that step time and step width variability are associated with different components of walking, namely rhythmic stepping pattern (i.e., progression) and balance control (i.e., walking stability), respectively (Gabell and Nayak 1984; Brach et al. 2005; Hausdorff 2005; Montero-Odasso et al. 2011). In this framework, our results suggested that consuming attentional resources with dichotic listening affected balance control similarly in both young and older adults, whereas it also altered control of rhythmic stepping pattern in the older adults. Therefore, the effect of aging seems to be expressed through a dual-task cost that extends from the balance-related gait characteristic to the stepping-related gait characteristic. This effect is likely a direct consequence of the declined attentional reserve capacity that occurs with advancing age (Lindenberger and Baltes 1994), which increases competition for attention resources between gait and the secondary cognitive task. However, the absence of a further increase in step width variability as a function of the attentional demand in the older adults is a bit surprising from the reserve capacity perspective. A possible explanation for this null finding may be that a more pronounced increase in step width variability could have led to incident mobility (e.g., falls) in the older adults, excessive step width variability having previously been found to be associated with a fall history (Brach et al. 2005). Alternatively, the older adults included in the study were healthy, which may have lessened the effects of attentional resource competition on balance control during gait. Examining gait-related dual-task effects in vulnerable older adults, such as individuals with a frailty phenotype or at risk of falling, using a protocol similar to the present study, may help address these issues.

With respect to cognitive performance, the older adults performed worse than the young adults on dichotic listening both in single-task and dual-task conditions. First, the older adults made more errors than their younger counterparts. Second, although both groups showed a REA in the NF condition, only the young adults were able to magnify and tone down this advantage in the FR and FL conditions, respectively. Overall, these results suggest a reduced capacity for cognitive control in older adults, which is consistent with previous studies (Andersson et al. 2008; Redfern et al. 2009). On the other hand, we found an unexpected result regarding the number of errors. The young adults made more errors while walking than while seated, indicating a dual-task cost in the cognitive domain. Inversely, there was a dual-task gain in the older adults with fewer errors while walking. Some previous studies already showed increased cognitive performance when walking was performed simultaneously with a concurrent cognitive task (Decker et al. 2012; Schaefer et al. 2010; Verrel et al. 2009). This effect was interpreted in terms of a general increase in arousal induced by physical activity. Similarly, walking may have boosted arousal in the older adults and positively affected their dichotic listening performance. That being said, the dual-task related changes in cognitive performance in young and older adults should not be overinterpreted. Indeed, the laterality index, which is a very common indicator of dichotic listening performance (Hugdahl et al. 2009), was not affected by the dual task. Accordingly, a cautious conclusion is that performance on the cognitive task was rather preserved under dualtask conditions in both groups.

The fact that the cognitive performance remained practically equivalent under both single- and dual-task conditions is important with respect to the task prioritization strategy used by the subjects. Indeed, a decreased gait control (i.e., increased step variability) combined with a preserved cognitive performance under dual-task conditions reflects a "posture second" strategy, where the cognitive task, and thus the attentional resources allocated to 
it, is prioritized over the gait task (Bloem et al. 2006; Yogev-Seligmann et al. 2012a, b). Accordingly, our data are in favor of such a strategy in both groups, providing further support to the idea that healthy adults, young and older, do not use "posture first" as a default strategy (Yogev-Seligmann et al. 2010, 2012b). The "posture second" strategy could be due to either an impaired judgment of the individuals with respect to the risk of their actions, exposing themselves to the risk of falling, as in patients with neurodegenerative diseases characterized by a cognitive decline (Bloem et al. 2006), or a high postural reserve that enables the individuals to focus on the cognitive task even when the gait threat is high (Bloem et al. 2006; Yogev-Seligmann et al. 2010, 2012a, b; Holtzer et al. 2014). Given that our subjects were cognitively intact, we propose that a high postural reserve was responsible for the "posture second" strategy they used. In fact, the gait task was fairly easy (i.e., at preferred walking speed), our subjects did not feel sufficiently threatened regarding balance and gait control to be in need of a "posture first" strategy. If the gait task was a little closer to their postural reserve capacity, they might have preferred to attend more their gait. The important point here is that the preference in preserving the cognitive task over gait control undoubtedly magnified the effects of attentional resource competition on gait with increasing cognitive task difficulty (i.e., the rising part of the Ushaped relationship). Indeed, a posture first strategy could have prevented observation of gait control decrements due to attentional resource competition (increased type II error, Verghese et al. 2007). Therefore, interpreting dualtask performance within the reserve capacity view should be systematically carried out in relation with the prioritization strategy used by the subjects.

This study is limited by the moderate sample size and the relative homogeneity of the older adults, all presenting a good functional status. Accordingly, some findings, such as the absence of further decrements in balance control when increasing the attentional demand and the prioritization of the cognitive task over the gait task, may not be generalizable to all community-dwelling older adults. Therefore, reproduction of the present findings in a larger sample, including also older persons with lower functionality, is warranted. Furthermore, we used treadmill walking instead of overground walking to produce robust estimates of step variability. The corollary is that treadmill walking has been found to be associated with a reduction of variability (Dingwell et al. 2001), which may have masked some dual-task related gait changes as previously mentioned.

In conclusion, our study provided evidence for a Ushaped relationship between cognitive demand and step variability, presumably indicating a trade-off between an external focus of attention and a competition for attentional resources. Thus, it appears appropriate to interpret the dual-task walking findings from young adulthood to old age using this dual-process model framework. Furthermore, the fact that the generalizability of the model to step parameters was not only related to balance control (step width variability) but also to rhythmic stepping pattern (step time variability) in older adults reveals that attentional resources become involved in all facets of walking with aging. Finally, our findings suggest that prioritizing the cognitive task over the gait task (i.e., a posture second strategy) may constitute the default strategy used by healthy individuals in dual-task walking. Strategies of task prioritization are also important to consider when interpreting the effects of dual tasking on gait.

Acknowledgments This study was supported by the National Institute on Aging at the National Institutes of Health (1K99AG033684). The authors are very grateful to Sharon Lynn Salhi, Ph.D., for presubmission editorial assistance.

\section{References}

Andersson M, Reinvang I, Wehling E, Hugdhal K, Lundervold AJ (2008) A dichotic listening study of attention control in older adults. Scand J Psychol 49:299-304

Asbjørnsen A, Hugdahl K (1995) Attentional effects in dichotic listening. Brain Lang 49:189-201

Asbjørnsen A, Holmefjord A, Reisaeter S, Møller P, Klausen O, Prytz B, Boliek C, Obrzut JE (2000) Lasting auditory attention impairment after persistent middle ear infections: a dichotic listening study. Dev Med Child Neurol 42:481-486

Aylward GP, Gordon M, Verhulst SJ (1997) Relationships between continuous performance task scores and other cognitive measures: causality or commonality. Assessment 4:325-336

Beauchet O, Dubost V, Aminian K, Gonthier R, Kressig RW (2005a) Dual-task-related gait changes in the elderly: does the type of cognitive task matter? J Mot Behav 37:259-264

Beauchet O, Dubost V, Herrmann FR, Kressig RW (2005b) Strideto-stride variability while backward counting among healthy young adults. J Neuroeng Rehabil 2:26

Bloem BR, Grimbergen YA, van Dijk JG, Munneke M (2006) The "posture second" strategy: a review of wrong priorities in Parkinson's disease. J Neurol Sci 248:196-204

Bouma A, Gootjes L (2011) Effects of attention on dichotic listening in elderly and patients with dementia of the Alzheimer type. Brain Cogn 76:286-293. doi:10.1016/j. bandc.2011.02.008 
Brach JS, Berlin JE, VanSwearingen JM, Newman AB, Studenski SA (2005) Too much or too little step width variability is associated with a fall history in older persons who walk at or near normal gait speed. J Neuroeng Rehabil 2:21. doi:10.1186/1743-0003-2-21

Braver TS, Cohen JD (2000) On the control of control: the role of dopamine in regulating prefrontal function and working memory. In: Monsell S, Driver J (eds) Attention and performance XVIII. MIT Press, Cambridge, MA, pp 713-737

Braver TS, Cohen JD, Barch DM (2002) The role of prefrontal cortex in normal and disordered cognitive control: a cognitive neuroscience perspective. In: Stuss DT, Knight RT (eds) Principles of frontal lobe function. Oxford University Press, New York, pp 428-448

Bryden MP (1988) An overview of the dichotic listening procedure and its relation to cerebral organization. In: Hugdahl K (ed) Handbook of dichotic listening: theory, methods, and research. Wiley \& Sons, Chichester, UK, pp 1-44

Bryden MP, Munhall K, Allard F (1983) Attentional biases and the right-ear effect in dichotic listening. Brain Lang 18:236-248

Bush G, Luu P, Posner MI (2000) Cognitive and emotional influences in the anterior cingulate cortex. Trends Cogn Sci 4: 215-222

Buzzi UH, Stergiou N, Kurz MJ, Hageman PA, Heidel J (2003) Nonlinear dynamics indicates aging affects variability during gait. Clin Biomech 18:435-443

Cherry EC (1953) Some experiments on the recognition of speech, with one and with two ears. J Acoust Soc Am 25:975-979

Cignetti F, Decker LM, Stergiou N (2012) Sensitivity of the Wolf's and Rosenstein's algorithms to evaluate local dynamic stability from small gait data sets: response to commentaries by Bruijn et al. Ann Biomed Eng 40:2507-2509. doi:10.1007 /s10439-012-0665-6

Decker LM, Cignetti F, Potter JF, Studenski SA, Stergiou N (2012) Use of motor abundance in young and older adults during dual-task treadmill walking. PLoS One 7, e41306. doi:10.1371/journal.pone.0041306

Decker LM, Cignetti F, Stergiou N (2013) Executive function orchestrates regulation of task-relevant gait fluctuations. Gait Posture 38:537-540. doi:10.1016/j.gaitpost.2012.12.018

Della Penna S, Brancucci A, Babiloni C, Franciotti R, Pizzella V, Rossi D, Torquati K, Rossini PM, Romani GL (2007) Lateralization of dichotic speech stimuli is based on specific auditory pathway interactions: neuromagnetic evidence. Cereb Cortex 17:2303-2311

Diamond A (2013) Executive functions. Annu Rev Psychol 64: 135-168. doi:10.1146/annurev-psych-113011-143750

Dingwell JB, Cusumano JP (2015) Identifying stride-to-stride control strategies in human treadmill walking. PLoS One 10, e0124879. doi:10.1371/journal.pone.0124879

Dingwell JB, Cusumano JP, Cavanagh PR, Sternad D (2001) Local dynamic stability versus kinematic variability of continuous overground and treadmill walking. J Biomech Eng $123: 27-32$

Dingwell JB, John J, Cusumano JP (2010) Do humans optimally exploit redundancy to control step variability in walking? PLoS Comput Biol 6, e1000856. doi:10.1371/journal. pcbi. 1000856

Dubost V, Kressig RW, Gonthier R, Herrmann FR, Aminian K, Najafi B, Beauchet O (2006) Relationships between dual-task related changes in stride velocity and stride time variability in healthy older adults. Hum Movement Sci 25:372-382

Dubost V, Annweiler C, Aminian K, Najafi B, Herrmann FR, Beauchet O (2008) Stride-to-stride variability while enumerating animal names among healthy young adults: result of stride velocity or effect of attention-demanding task? Gait Posture 27:138-143

Folstein MF, Folstein SE, McHugh PR (1975) "Mini-mental state". A practical method for grading the cognitive state of patients for the clinician. J Psychiatr Res 12:189-198

Foundas AL, Corey DM, Hurley MM, Heilman KM (2006) Verbal dichotic listening in right and left-handed adults: laterality effects of directed attention. Cortex 42:79-86

Gabell A, Nayak US (1984) The effect of age on variability in gait. J Gerontol 39:662-666

Grabiner MD, Troy KL (2005) Attention demanding tasks during treadmill walking reduce step width variability in young adults. J Neuroeng Rehabil 2:25

Griggs RA, Platt RD, Newstead SE, Jackson SL (1998) Attentional factors in a disjunctive reasoning task. Think Reason 4:1-14

Hausdorff JM (2005) Gait variability: methods, modeling and meaning. J Neuroeng Rehabil 2:19

Hausdorff JM, Rios DA, Edelberg HK (2001) Gait variability and fall risk in community-living older adults: a 1-year prospective study. Arch Phys Med Rehabil 82:1050-1056

Herman T, Mirelman A, Giladi N, Schweiger A, Hausdorff JM (2010) Executive control deficits as a prodrome to falls in healthy older adults: a prospective study linking thinking, walking, and falling. J Gerontol A Biol Sci Med Sci 65: 1086-1092. doi:10.1093/gerona/glq077

Hill KD, Schwarz JA, Kalogeropoulos AJ, Gibson SJ (1996) Fear of falling revisited. Arch Phys Med Rehabil 77:1025-1029

Hollman JH, Kovash FM, Kubik JJ, Linbo RA (2007) Age-related differences in spatiotemporal markers of gait stability during dual task walking. Gait Posture 26:113-119

Holtzer R, Wang C, Verghese J (2014) Performance variance on walking while talking tasks: theory, findings, and clinical implications. Age (Dordr) 36:373-381. doi:10.1007 /s11357-013-9570-7

Hugdahl K (2003) Dichotic listening in the study of auditory laterality. In: Hugdahl K, Davidson RJ (eds) The asymmetrical brain. MIT Press, Cambridge, MA, pp 441-476

Hugdahl K, Andersson L (1986) The "forced-attention paradigm" in dichotic listening to CV-syllables: a comparison between adults and children. Cortex 22:417-432

Hugdahl K, Westerhausen R, Alho K, Medvedev S, Laine M, Hämäläinen H (2009) Attention and cognitive control: unfolding the dichotic listening story. Scand J Psychol 50: 11-22. doi:10.1111/j.1467-9450.2008.00676.x

Hunt E, Lansman M (1986) Unified model of attention and problem solving. Psychol Rev 93:446-461

Huxhold O, Li SC, Schmiedek F, Lindenberger U (2006) Dual tasking postural control. Aging and the effects of cognitive demand in conjunction with focus of attention. Brain Res Bull 69:294-305

Jordan K, Challis JH, Newell KM (2007) Walking speed influences on gait cycle variability. Gait Posture 26:128-134

Kang HG, Dingwell JB (2008) Separating the effects of age and walking speed on gait variability. Gait Posture 27:572-577 
Kimura D (1961) Cerebral dominance and the perception of verbal stimuli. Can J Psychol 15:166-171

Kinsbourne M (1970) The cerebral basis of lateral asymmetries in attention. Acta Psychol (Amst) 33:193-201

Kinsbourne M (1975) The mechanism of hemispheric control of the lateral gradient of attention. In: Rabbitt PMA, Dornic S (eds) Attention and performance V. Academic, London, New York

Kressig RW, Herrmann FR, Grandjean R, Michel JP, Beauchet O (2008) Gait variability while dual-tasking: fall predictor in older inpatients? Aging Clin Exp Res 20:123-130

Li KZ, Lindenberger U (2002) Relations between aging sensory/ sensorimotor and cognitive functions. Neurosci Biobehav Rev 26:777-783

Lindenberger U, Baltes PB (1994) Sensory functioning and intelligence in old age: a strong connection. Psychol Aging 9: 339-355

Lövdén M, Schaefer S, Pohlmeyer AE, Lindenberger U (2008) Walking variability and working memory load in aging: a dual-process account relating cognitive control to motor control performance. J Gerontol B Psychol Sci Soc Sci 63:P121-P128

Miller EK, Cohen JD (2001) An integrative theory of prefrontal cortex function. Annu Rev Neurosci 24:167-212

Monaco M, Costa A, Caltagirone C, Carlesimo GA (2013) Forward and backward span for verbal and visuo-spatial data: standardization and normative data from an Italian adult population. Neurol Sci 34:749-754. doi:10.1007/s10072012-1130-x

Montero-Odasso M, Muir SW, Hall M, Doherty TJ, Kloseck M, Beauchet O, Speechley M (2011) Gait variability is associated with frailty in community-dwelling older adults. J Gerontol A Biol Sci Med Sci 66:568-576. doi:10.1093 /gerona/glr007

Newell KM, Corcos DM (1993) Issues in variability and motor control. In: Newell KM, Corcos DM (eds) Variability and motor control. Human Kinetics, Champaign, IL, pp 1-12

Nigg BM, Cole GK, Nachbauer W (1993) Effects of arch height of the foot on angular motion of the lower extremities in running. J Biomech 26:909-916

Oldfield RC (1971) The assessment and analysis of handedness: the Edinburgh inventory. Neuropsychologia 9:97-113

Owings TM, Grabiner MD (2004) Variability of step kinematics in young and older adults. Gait Posture 20:26-29

Redfern MS, Jennings JR, Martin C, Furman JM (2001) Attention influences sensory integration for postural control in older adults. Gait Posture 14:211-216

Redfern MS, Jennings JR, Mendelson D, Nebes RD (2009) Perceptual inhibition is associated with sensory integration in standing postural control among older adults. J Gerontol B Psychol Sci Soc Sci 64:P569-P576. doi:10.1093 /geronb/gbp060

Schaefer S, Huxhold O, Lindenberger U (2006) Healthy mind in healthy body? A review of sensorimotor-cognitive interdependencies in old age. Eur Rev Aging Phys Act 3:45-54

Schaefer S, Lövdén M, Wieckhorst B, Lindenberger U (2010) Cognitive performance is improved while walking: differences in cognitive-sensorimotor couplings between children and young adults. Eur J Dev Psychol 7:371-389

Schaefer S, Jagenow D, Verrel J, Lindenberger U (2015) The influence of cognitive load and walking speed on gait regularity in children and young adults. Gait Posture 41:258-262. doi:10.1016/j.gaitpost.2014.10.013

Seidler RD, Bernard JA, Burutolu TB, Fling BW, Gordon MT, Gwin JT, Kwak Y, Lipps DB (2010) Motor control and aging: links to age-related brain structural, functional, and biochemical effects. Neurosci Biobehav Rev 34:721-733. doi:10.1016/j.neubiorev.2009.10.005

Sheikh JI, Yesavage JA (1986) Geriatric Depression Scale (GDS) (1986): recent evidence and development of a shorter version. In: Brink TL (ed) Clinical gerontology: a guide to assessment and intervention. The Haworth Press, Binghamton, NY, pp $165-173$

Springer S, Giladi N, Peretz C, Yogev G, Simon ES, Hausdorff JM (2006) Dual-tasking effects on gait variability: the role of aging, falls, and executive function. Movement Disord 21: 950-957

Stroop JR (1935) Studies of interference in serial verbal reactions. J Exp Psychol 18:643-661

Tervaniemi M, Hugdahl K (2003) Lateralization of auditorycortex functions. Brain Res Brain Res Rev 43:231-246

Thomsen T, Rimol LM, Ersland L, Hugdahl K (2004) Dichotic listening reveals functional specificity in prefrontal cortex: an fMRI study. Neuroimage 21:211-218

Vaughan C, Davis B, O’Connor J (1999) Dynamics of human gait. Kiboho Publishers, Cape Town, South Africa

Verghese J, Kuslansky G, Holtzer R, Katz M, Xue X, Buschke H, Pahor M (2007) Walking while talking: effect of task prioritization in the elderly. Arch Phys Med Rehabil 88:50-53

Verghese J, Holtzer R, Lipton RB, Wang C (2009) Quantitative gait markers and incident fall risk in older adults. J Gerontol Med Sci 64:896-901. doi:10.1093/gerona/glp033

Verrel J, Lövdén M, Schellenbach M, Schaefer S, Lindenberger U (2009) Interacting effects of cognitive load and adult age on the regularity of whole-body motion during treadmill walking. Psychol Aging 24:75-81. doi:10.1037/a0014272

Wechsler D (1981) Wechsler Adult Intelligence Scale-3rd edition. Psychological Corporation, San Antonio, TX

Yogev G, Giladi N, Peretz C, Springer S, Simon ES, Hausdorff JM (2005) Dual tasking, gait rhythmicity, and Parkinson's disease: which aspects of gait are attention demanding? Eur J Neurosci 22:1248-1256

Yogev-Seligmann G, Rotem-Galili Y, Mirelman A, Dickstein R, Giladi N, Hausdorff JM (2010) How does explicit prioritization alter walking during dual-task performance? Effects of age and sex on gait speed and variability. Phys Ther 90:177186. doi:10.2522/ptj.20090043

Yogev-Seligmann G, Hausdorff JM, Giladi N (2012a) Do we always prioritize balance when walking? Towards an integrated model of task prioritization. Mov Disord 27:765-770. doi: $10.1002 / \mathrm{mds} .24963$

Yogev-Seligmann G, Rotem-Galili Y, Dickstein R, Giladi N, Hausdorff JM (2012b) Effects of explicit prioritization on dual task walking in patients with Parkinson's disease. Gait Posture 35:641-646. doi:10.1016/j.gaitpost.2011.12.016 